\section{AD target gets a caution}

\section{By Lev Osherovich, Senior Writer}

A Harvard researcher says three new mouse studies reinforce her hypothesis that targeting $\gamma$-secretase to treat Alzheimer's disease could exacerbate, rather than ameliorate, $\mathrm{AD}$-related neuron loss and brain dysfunction. ${ }^{1-3}$ One neurology company targeting $\gamma$-secretase, Elan Corp. plc, has not been convinced.

$\gamma$-Secretase is an enzymatic complex that digests neuronal membrane proteins and is thought to cleave amyloid- $\beta$ (A4) precursor protein (APP) to generate the $\beta$-amyloid $(A \beta)$ fragments that many researchers believe cause $\mathrm{AD}$. But the new studies suggest that various components of the $\gamma$-secretase complex are essential for normal neuronal function and survival.

According to Jie Shen, senior author of two of the studies, these and earlier human genetic findings suggest that $\mathrm{AD}$ is caused by inadequate $\gamma$-secretase activity-not by A $\beta$ fragments-and that inhibiting the enzyme will only make things worse.

"In my mind, this is why $\gamma$-secretase is dead as a therapeutic target," she said. Shen, an associate professor of neurology at Harvard Medical School and Brigham and Women's Hospital, reported her results in The Journal of Neuroscience ${ }^{1}$ and Nature. ${ }^{2}$

\section{AD without the $A \beta$}

In The Journal of Neuroscience study, Shen's team shut off the expression of nicastrin (Ncstn) - one of the four subunits of $\gamma$-secretase-in mouse forebrains, the main area affected by AD.

When Ncstn expression was turned off during adulthood, $\gamma$-secretase activity decreased and the mice had age-dependent losses in neurons in the neocortex and hippocampus compared with wild-type controls.

Older nicastrin-deficient mice had impaired memory and cognition compared with wild-type controls. The defects mirrored those seen in other AD models but were not accompanied by higher levels of $A \beta$.

"We saw a recapitulation of essentially all the features of AD without the amyloid," said Shen. Thus, in contrast to most researchers in the field, she believes $\mathrm{A} \beta$ is irrelevant to $\mathrm{AD}$ pathogenesis. Instead, Shen thinks the problem may be inadequate levels of $\gamma$-secretase components.

Shen's subsequent Nature paper described more evidence to support her hypothesis. In that study, her team used an in vitro assay of neuronal function to examine the effect of knocking out two other $\gamma$-secretase components-presenilin 1 (PSEN1; PS1) and PSEN2 (PS2) - in various parts of the hippocampus.

The team found that when the presenilin genes were disrupted in the presynaptic neurons of the hippocampus, the cells proved less responsive to incoming signals and released lower levels of neurotransmitters than genetically normal neurons.

Based on the genetics of presenilin function, "impaired neurotransmitter release may be the earliest step in AD," said Shen.

Roberto Malinow, professor of neurobiology at the University of California, San Diego, said Shen's Nature study is a "nice demonstration that presynaptic presenilins are required for normal presynaptic function."

To clearly show a connection between presenilin deficiency and AD, Malinow said, Shen's team must next test the hippocampal PS1 and PS2 knockouts for age-related behavioral problems and neurodegeneration.

Dale Schenk, EVP and CSO of Elan, said Shen's studies may have uncovered additional roles for $\gamma$-secretase subunits but argued that discounting the effect of $\gamma$-secretase in creating the $A \beta$ fragments found in $\mathrm{AD}$ paints an incomplete picture of the disorder.

" $\gamma$-Secretase is a very complex enzyme," he said. "It has many functions including cleaving APP into $A \beta$, but this doesn't preclude its importance in other neuronal functions."

Elan's ELND-006, a $\gamma$-secretase modulator, is in Phase I testing for AD. Last month, Elan and Johnson \& Johnson said they will form a new company to work on an AD immunotherapy program, which Elan has partnered with Wyeth. The program includes four products targeting $A \beta$, including bapineuzumab, a Phase III mAb.

The most advanced $\gamma$-secretase inhibitor, semagacestat (LY450139) from Eli Lilly and Co., is in Phase III testing for AD.

Schenk thinks the complete disruption of $\gamma$-secretase activity caused by Shen's knockout experiments causes a more severe effect than is observed in familial $\mathrm{AD}$, which involves more subtle alterations to $\gamma$-secretase components. He thus thinks that partial inhibition of $\gamma$-secretase with next-generation modulators may block production of $A \beta$ without causing the major neurological defects found in Shen's knockouts.

"With a knockout, it's all or none, but in medicine we are titrating a drug to achieve a therapeutic effect," said Schenk.

Shen thinks the true test of her idea will be to make transgenic mice expressing human versions of presenilins bearing AD-linked mutations. If her theory is correct, the transgenic mice should have $\mathrm{AD}$-like phenotypes but normal $A \beta$ levels-just like the selective presenilin and nicastrin knockouts.

The mice will play

A team at Institut National de la Santé et de la Recherche Médicale (INSERM) has already generated a knock-in mouse that overexpresses 


\section{TARGETS \& MECHANISMS}

human PS1 with an AD-associated mutation.

The French team found that the mutant PS1 caused an age-related decline in neuronal function, with mice having lower sensitivity to hippocampal stimulus and less neurotransmission than wild-type controls. However, the group did not observe neurodegeneration, suggesting that high levels of mutant PS1 can interfere with normal neuronal activity without killing the cells.

According to the authors, the study adds to emerging evidence of "other possible effects of PS1 on synaptic dysfunction where PS1 might contribute to the pathology independent of $A \beta$."

Jean Mariani, professor of neurobiology at INSERM, was the team leader, and results were published in The Journal of Neuroscience. ${ }^{3}$

Shen noted that although the Mariani study "sheds light on the disease process," the findings are complicated by the presence of murine Ps1 in the strain used by the French team. However, it should be straightforward to knock out the native mouse gene in Mariani's PS1 transgenic strain, which would allow Shen to test her hypothesis.

Although Shen is convinced that blocking $\gamma$-secretase is a bad idea, it remains unclear why $\gamma$-secretase or its subunits are needed for neuronal survival. One possibility is that besides converting APP to A $\beta$, $\gamma$-secretase may process unidentified proteins that promote neuronal survival. Alternatively, the enzyme's subunits may have additional roles in the cell unrelated to $\gamma$-secretase.

Shen believes that, in addition to being part of the $\gamma$-secretase complex, presenilins play a role in calcium homeostasis and intracellular signaling within neurons, and that these functions may be compromised in AD.

For example, Shen's team reported in the Nature article that hippocampal presenilin knockouts had lower levels of intracellular calcium than wild-type controls. This was due in part to inactivation of ryano- dine receptors, which are internal relays for neuronal activation.

Shen has patented the idea of targeting ryanodine receptors to treat $\mathrm{AD}$ and plans to explore how these proteins interact with presenilins.

"Our idea is that the ryanodine receptor is impaired in $\mathrm{AD}$ and an agent to reverse this impairment may be therapeutic," she said.

Osherovich, L. SciBX 2(31); doi:10.1038/scibx.2009.1201

Published online Aug. 13, 2009

\section{REFERENCES}

1. Tabuchi, K. et al. J. Neurosci.; published online June 3, 2009; doi:10.1523/JNEUROSCI.1320-09.2009

Contact: Jie Shen, Harvard Medical School, Boston, Mass. e-mail: jshen@rics.bwh.harvard.edu

2. Zhang, C. et al. Nature; published online July 30, 2009; doi:10.1038/nature08177

Contact: Jie Shen, Harvard Medical School, Boston, Mass. e-mail: jshen@rics.bwh.harvard.edu

Contact: Chen Zhang, same affiliation as above e-mail: czhang@rics.bwh.harvard.edu

3. Auffret, A. et al. J. Neurosci.; published online Aug. 12, 2009; doi:10.1523/JNEUROSCI.1856-09.2009

Contact: Alexandra Auffret, University of Pierre and Marie Curie, Paris, France

e-mail: alexandraauffret@gmail.com

COMPANIES AND INSTITUTIONS MENTIONED

Brigham and Women's Hospital, Boston, Mass.

Elan Corp. plc (NYSE:ELN), Dublin, Ireland

Eli Lilly and Co. (NYSE:LLY), Indianapolis, Ind.

Harvard Medical School, Boston, Mass. Institut National de la Santé et de la Recherche Médicale, Paris, France

Johnson \& Johnson (NYSE:JNJ), New Brunswick, N.J. University of California, San Diego, La Jolla, Calif. Wyeth (NYSE:WYE), Madison, N.J. 